\title{
Improving medication adherence with adjuvant aromatase inhibitor in women with breast cancer: study protocol of a randomised controlled trial to evaluate the effect of short message service (SMS) reminder
}

Yunxin He ${ }^{1}$, Eng Hooi Tan ${ }^{1}$, Andrea Li Ann Wong ${ }^{2}$, Chuan Chien Tan ${ }^{3}$, Patrick Wong ${ }^{4}$, Soo Chin Lee ${ }^{2}$ and Bee Choo Tai ${ }^{1,5^{*}}$

\begin{abstract}
Background: Medication adherence refers to whether a patient takes medication according to the frequency prescribed, or continues to take a prescribed medication. Inadequate adherence to medication may cause alterations in risk-benefit ratios, resulting in reduced benefits, increased risks or both, and is significantly associated with adverse clinical outcomes and higher healthcare costs. We aim to examine the effect of a computer generated short message service (SMS) reminder in improving medication adherence, and inhibiting the aromatisation process amongst breast cancer women receiving oral aromatase inhibitor therapy.

Methods/Design: In this randomised controlled trial, eligible patients will be equally allocated to receive either SMS reminder or standard care. The former receives weekly SMS reminder to take medication while the latter does not receive any. The primary endpoint of medication adherence at 1-year is assessed using the Simplified Medication Adherence Questionnaire, and compared using the $x^{2}$ test. Adjustment for baseline covariate and potential confounders will be made using the logistic regression. Secondary outcomes involving estrone and androstenedione levels will be compared using the analysis of covariance, whereas the estradiol levels $(<18$. $4 \mathrm{pmol} / \mathrm{L}$ versus $\geq 18.4 \mathrm{pmol} / \mathrm{L}$ ) will be compared using the $X^{2}$ test, and the logistic regression. Further, the assessment of knowledge, attitude, behaviour, and barriers and facilitating factors of medication adherence will be made via logistic regression.

* Correspondence: ephtbc@nus.edu.sg

'Saw Swee Hock School of Public Health, National University of Singapore and National University Health System, 12 Science Drive 2, \#10-03F,

Singapore 117549, Singapore

${ }^{5}$ Yong Loo Lin School of Medicine, National University of Singapore and

National University Health System, 1E Kent Ridge Road, Singapore 119228,

Singapore

Full list of author information is available at the end of the article

(c) The Author(s). 2018 Open Access This article is distributed under the terms of the Creative Commons Attribution 4.0 International License (http://creativecommons.org/licenses/by/4.0/), which permits unrestricted use, distribution, and reproduction in any medium, provided you give appropriate credit to the original author(s) and the source, provide a link to the Creative Commons license, and indicate if changes were made. The Creative Commons Public Domain Dedication waiver (http://creativecommons.org/publicdomain/zero/1.0/) applies to the data made available in this article, unless otherwise stated. 
(Continued from previous page)

Discussion: This will be the first study to evaluate short-term clinical outcomes from SMS reminder for breast cancer patients on aromatase inhibitor therapy. Random allocation to SMS reminder or control arm ensures that patients in both arms will be comparable with respect to demographic and clinical characteristics, and any difference in outcomes can be attributed to the intervention. Participants are not blinded to the assignment of intervention, thus there may be potential for bias in outcome assessments.

Trial registration: NCT02524548. Retrospectively registered on 17 August 2015.

Keywords: Randomised controlled trial, Aromatase inhibitor therapy, Breast cancer, Medication adherence, SMS reminder

\section{Background}

Medication adherence typically refers to whether a patient takes medication according to the frequency prescribed, or continues to take a prescribed medication [1]. The World Health Organisation defines medication adherence to long-term therapy as "the extent to which a person's behaviour - taking medication, following a diet, and/or executing lifestyle changes, corresponds with agreed recommendations from a health care provider" [2]. Accruing evidences show that inadequate medication compliance can cause alterations in benefit/risk ratios, resulting in reduced benefits, increased risks or both [3, 4]. Medication non-adherence is significantly associated with adverse clinical outcomes and higher healthcare costs [5].

\section{Aromatase inhibitor and breast cancer risk and recurrence}

Breast cancer is the leading type of cancer affecting women, and it was estimated that worldwide over 508,000 women died of this condition in 2011 [6]. In Singapore, it is also the primary cause of death for women from 2011 to 2015 [7]. Besides, a total of 9634 new cases of breast cancer were diagnosed in the same period, accounting for $29.1 \%$ of incident cancers in females and making it the most common cancer among women [7].

Estrogen promotes the growth and survival of normal and cancerous breast epithelial cells by binding and activating the estrogen receptor (ER). The activated receptor in turn binds to gene promoters in the nucleus and activates many other genes responsible for cell division, inhibition of cell death, new blood vessel formation and protease activity [8]. Thus, hormone therapy, which is a form of systemic therapy, is recommended for women with hormone receptor-positive breast cancer. Aromatase inhibitor (AI), a commonly prescribed hormonal therapy for early stage breast cancer, interferes with the body's ability to produce estrogen from androgens by suppressing the aromatase enzyme activity. Research has shown that women treated with adjuvant hormone therapy for early-stage ER positive breast cancer gain at least 5 more years due to the treatment.
However, it has been reported that patients could suffer from serious side effects of the hormone therapy [9].

Key et al. demonstrated a significant association between breast cancer risk and circulating estrogens in postmenopausal women [10]. Estrogen is the main stimulant in the development and growth of breast cancer, [11] and higher endogenous estrogen level has been found to be associated with elevated breast cancer risk. Tamoxifen has been successfully used for treating ER positive breast cancer for the past three decades. More recently, AI has been demonstrated to provide a significant reduction in breast cancer recurrence in post-menopausal women [4]. Following menopause, aromatase in fat and muscle may be responsible for much of the circulating estrogen. In highly estrogen-sensitive tissues such as the breast, uterus, vagina, bone, brain, heart and blood vessels, it provides local estrogen in an autocrine fashion. The aromatase gene promoter in breast tissue is less sensitive than the gene promoter in the ovary due to fluctuations in luteinising hormone but much more sensitive to increases in inflammatory cytokines, which increases with age. In particular, breast tissue inflammatory cytokines increase with proliferative breast disease and breast cancer [8].

A prospective, multi-centre, non-interventional study reported significant improvements in long-term quality of life of postmenopausal Chinese patients with hormonereceptor-positive early-stage breast cancer after starting treatment with AI [12]. A recent meta-analysis also showed the superiority of AI as an adjuvant hormonal therapy in improving the disease-free and overall survival of postmenopausal ER-positive breast cancer as compared to tamoxifen [13]. In another clinical trial, researchers have suggested that improvement in overall survival (hazard ratio 0.78 ; $95 \%$ CI 0.62 to 0.98 ) was seen among premenopausal women with the use of exemestane plus ovarian suppression as compared to tamoxifen users [14].

However, adverse events include hot flushes, vaginal dryness, loss of libido, fatigue, arthralgia, joint stiffness and loss of bone mineral density with subsequent increased risk of fracture $[8,15]$. Also, as the treatment of AI is long-term, medication adherence is an issue of concern, with a proportion of women stopping before 
completing the full treatment, while others not taking a daily tablet regularly $[16,17]$. Its adherence rate has been shown to decline over time from 78 to $86 \%$ in the first year, and reaching 62 to $79 \%$ by Year 3 [17]. A recent nested case-control study showed that non-adherence to oral endocrine therapy was significantly associated with worse breast cancer survival (OR 4.07; 95\% CI 3.27-5.06) [18].

\section{Intervention to improve medication adherence}

Recent reviews have shown that although various behavioural, educational, integrated care and self-management risk communication interventions have been implemented to improve medication adherence, none of them have shown promising impact $[19,20]$. On the other hand, studies have suggested that reminder of any form, such as setting an alarm on a regular daily basis at home, or have family reminding the patient to take medication, have a positive influence on medication adherence in cancer patients [19]. While healthcare institutions sometimes use short message service (SMS) to remind patients of follow-up clinic appointments, mobile technology is seldom implemented to monitor medication adherence until the recent decade [21]. This is a feasible and acceptable form of managing medication which has a high participant satisfaction [22]. Its use has been shown to improve medication adherence in cardiovascular disease, diabetes, HIV, oral contraception, asthma, as well as oncology patients with chronic conditions $[22,23]$. As mobile phone ownership continues to increase, there is a great potential to utilise this technology to overcome adherence barriers and optimise therapeutic effects [24].

\section{Barriers and facilitating factors influencing medication adherence}

Malcolm et al. identified cost, side-effects, transportation, lack of reimbursement for the medication and inefficient patient-physician communication as barriers to medication adherence [25]. Medication-taking behaviour has been shown to be influenced by a patient's belief and his/her trust in the physician who prescribes the medication [26]. Also, non-adherence to endocrine therapy might be due to patients' response to the AI therapy and its associated side effects such as arthralgia [27]. Besides, it has been shown that nonadherence to medications for chronic diseases prior to hormonal therapy was associated with more severe nonadherence to oral hormonal therapy in patients with breast cancer [28] A cross-sectional study comprising mostly White women showed that survivors who perceived that their pain made taking AIs difficult or that the AI treatment lasted too long were likely to be non-adherent, as assessed by the Health Beliefs Scale Items and Scale Properties [29]. In contrast, a systematic review showed that taking more medications at baseline, referral to an oncologist, and being diagnosed at earlier times were positively associated with adherence and/or persistence [16]. Thus, in non-adherent patients, it is desirable to understand their attitude and perception towards medication taking.

Cancer treatment adherence plays a crucial role in optimising health outcomes while medication non-adherence is associated with decreased survival, higher recurrences and increased healthcare costs. Hsieh et al. reported that interruption and non-adherence to long-term adjuvant hormone therapy was associated with increased mortality in breast cancer women [30]. Treatment non-adherence to adjuvant hormonal therapy was found to be associated with increased all-cause mortality amongst Asian breast cancer women. Moreover, a greater impact of non-adherence on mortality was especially found in the younger breast cancer population [30].

Implementing innovative technology to tackle the problem of non-adherence and understanding its barriers will provide scientific evidence for clinical decision making and improve health outcomes. We thus propose to examine the effect of a computer automated SMS reminder in improving medication adherence amongst breast cancer women receiving oral AI therapy.

\section{Study objectives}

The primary objective is to evaluate whether SMS reminder improves medication adherence as compared to standard care (control) amongst breast cancer women who have been receiving adjuvant endocrine therapy for at least 1 year, and are continuing to receive adjuvant $\mathrm{AI}$ therapy for at least 1 more year.

The secondary objectives are to examine whether SMS reminder improves the inhibition of aromatisation process of patients receiving AI therapy. We postulate that at 1-year, there will be

- Lower estrone and estradiol levels in the SMS reminder group as compared to the control.

- Higher androstenedione level in the SMS reminder group as compared to the control.

Tertiary objectives include

- Assessing the impact of SMS reminder on the improvement in knowledge, attitude and behaviour towards medication compliance.

- Identifying barriers and facilitating factors for medication adherence.

\section{Methods/Design}

\section{Study design}

This is an open-label, multi-centre prospective randomised controlled trial of SMS reminder versus standard 
care to investigate whether SMS reminder improves adherence to oral AI therapy in breast cancer women at 1-year follow-up. A total of 280 breast cancer women will be recruited from the National University Cancer Institute, Singapore and the $\mathrm{Ng}$ Teng Fong General Hospital, Singapore. Eligible participants will receive financial compensation for each study visit in an amount within the guidelines of the ethics review board.

\section{Inclusion criteria}

- Women who have been receiving adjuvant endocrine therapy for at least 1 year, and are continuing to receive adjuvant AI therapy for at least 1 more year.

- Aged 21 to 80 years.

- Have cellular phone that can receive text messages.

- Singaporean or permanent resident who is currently residing in Singapore.

- Able to give informed consent.

\section{Exclusion criteria}

- Unable or not willing to comply with study procedures.

\section{Study intervention}

A systematic review involving HIV patients found weekly reminders to significantly improve the percentage of achieving 90\% adherence, while daily reminders showed no improvement [31]. Thus, we propose to send weekly SMS reminders on the first working day of each week, that is, at 9:00 AM each Monday morning from a tablet via an automated messaging system, throughout the 1-year follow-up period for the intervention group. A screen shot of the message sent is shown in Fig. 1.

The message includes information on patient's name, date, hospital, medication and frequency of oral AI therapy. In order to cater to the multi-ethnic study population, the message is translated to Mandarin or Malay. If a patient is not proficient in English, the messages will be sent using her preferred language. The control group will not receive any message. Regardless of the randomised allocation, all patients will be given a log for the next 6 months to record whether they have received the message (for the intervention group) or taken the pills according to instruction. The log will be recalled on the subsequent visit and a new log will be distributed to patients for recording the information for the subsequent 6 months. This log will be returned to the study coordinator at the end of the study.

\section{Randomisation procedure}

All eligible patients who have provided informed consent will be randomised to receive either SMS reminder or standard care in a 1:1 ratio. Balanced permuted block randomisation will be implemented with varying block sizes, stratified by centre. Allocation concealment will be ensured, as the randomisation allocation, generated by the responsible statistician, will not be released to the research coordinator until the patient has been recruited into the trial by means of a central telephone call upon meeting the eligibility criteria. The study flow chart is shown in Fig. 2.

\section{Data collection}

Three questionnaires will be administered via face-to-face interview by the Research coordinator. The Simplified Medication Adherence Questionnaire (SMAQ) is a validated 6-item questionnaire which measures adherence [32]. Non-compliance is defined if a patient responds to any of items 1 to 4 with a non-adherence answer; and if the patient has skipped more than two doses during the last week or has not taken medication for more than two complete days during the last visit. In addition, information on knowledge, attitude and behaviour towards medication taking will be elicited via the Beliefs about Medicines Questionnaire (BMQ) [33] and the Adherence Starts with Knowledge (ASK-12) questionnaire [34].

\section{Follow-up and assessments}

The assessments and follow-up schedule (with a window period of \pm 2 months) are summarised in Table 1 .

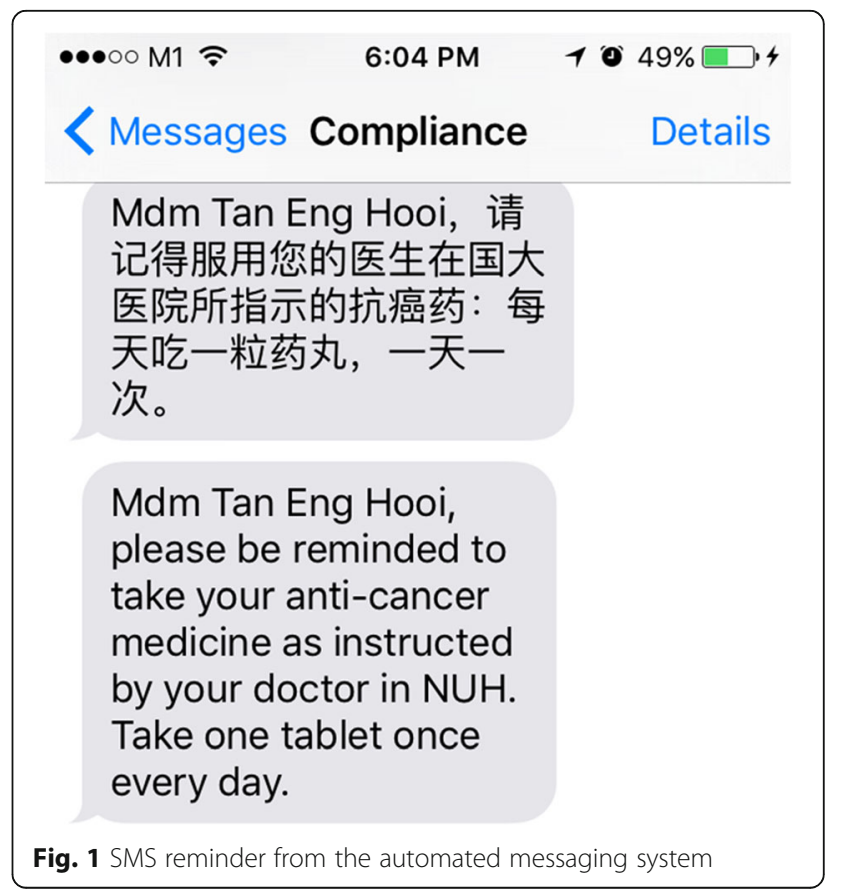


The patients will be followed up half yearly. The SMAQ will be administered at each visit, while the BMQ and ASK-12 questionnaires will be administered at baseline and 1-year. The hormone assays of estrone, estradiol and androstenedione will also be performed at baseline and 1-year.

This trial does not involve any experimental treatment and thus poses no additional risks to patients. Nevertheless, at each follow-up visit, the research coordinator will record any symptom or event that a patient may have experienced that is considered as UPIRTSO (Unanticipated Problems Involving Risks to Subjects and Others) event. These events will be monitored closely using a log book.

\section{Statistical considerations}

\section{Sample size}

The sample size is estimated based on the primary endpoint of medication adherence (as measured via SMAQ) at 1-year. Assuming that the proportion of medication adherence in the intervention and control groups are 80 and $60 \%$ respectively, [17] then based on a $5 \%$ significance level and a power of $90 \%$, a minimum sample of 240 subjects (i.e. 120 per group) will be required. Further assuming an attrition rate of $10 \%$, the overall trial size is 280 .

\section{Statistical analysis plan}

The assessment of medication adherence at 1-year between the SMS reminder and control groups will be made using the $\chi^{2}$ test, with adjustment for potential confounders made using the logistic regression.

Natural log transformation will be implemented on the estrone and androstenedione measures to normalise the data. The comparison of secondary outcomes involving estrone and androstenedione levels will be made using the analysis of covariance (ANCOVA) to adjust for the respective baseline levels and other potential confounders. The estradiol levels (defined as $<18.4 \mathrm{pmol} / \mathrm{L}$ versus $\geq 18.4 \mathrm{pmol} / \mathrm{L}$ ) will be compared between the two arms using the $\chi^{2}$ test and the logistic regression analysis will be implemented to adjust for baseline covariate and other potential confounders where appropriate.

The assessment of knowledge, attitude, behaviour, as well as barrier and facilitating factors between the intervention and control groups will be made using $\chi^{2}$ tests, with treatment effect quantified based on odds ratio estimate and its 95\% confidence interval. Further adjustment for baseline scores and other potential confounders will be made using the logistic regression analysis where appropriate.

All analyses will be performed according to the principle of intention-to-treat using STATA version 15, assuming a two-sided test at the $5 \%$ level of significance.

\section{Assessing medication adherence}

There are different ways of assessing medication compliance. Patient self-reporting relies mainly on a patient's recall, and is susceptible to bias. Pill count has also been shown to be unreliable especially if patients fail to return the bottles or dumped the pills before the count [35]. Medication possession ratio is commonly used for claims-based adherence measurement. However, it overestimates the true adherence rate particularly when a patient receives an early refill of the medication. The proportion of days covered (PDC), a recent method for calculating adherence, avoids double counting when refills overlap or when oversupply of medication exists [36]. However, it ignores instances when patients refill their prescriptions before finishing the drug.

In a further sub-study, we will compare the four different measures of adherence, to determine which of these provide a more meaningful or reliable information in the local context based on different performance indicators

Table 1 Summary of study procedures and schedule

\begin{tabular}{|c|c|c|c|}
\hline \multirow[t]{2}{*}{ Study procedures } & Visit 1 & Visit 2 & Visit 3 \\
\hline & Screening/Baseline & Month 6 & Month 12 \\
\hline Informed consent & $x$ & & \\
\hline Assess eligibility & $x$ & & \\
\hline SMAQ & $x$ & $x$ & $x$ \\
\hline BMQ, ASK-12 & $x$ & & $x$ \\
\hline Prescription data & $x$ & $x$ & $x$ \\
\hline Pill count and refill data & & $x$ & $x$ \\
\hline Estrone, estradiol and androstenedione assays & $x$ & & $x$ \\
\hline UPIRTSO event & & $x$ & $x$ \\
\hline Patient log of daily pill taken and weekly SMS & & $x$ & $x$ \\
\hline${ }^{\mathrm{a}}$ Check receipt of SMS & $x$ & $x$ & $x$ \\
\hline
\end{tabular}

${ }^{a}$ Research coordinator will verbally check the status of SMS receipt at each clinic visit 


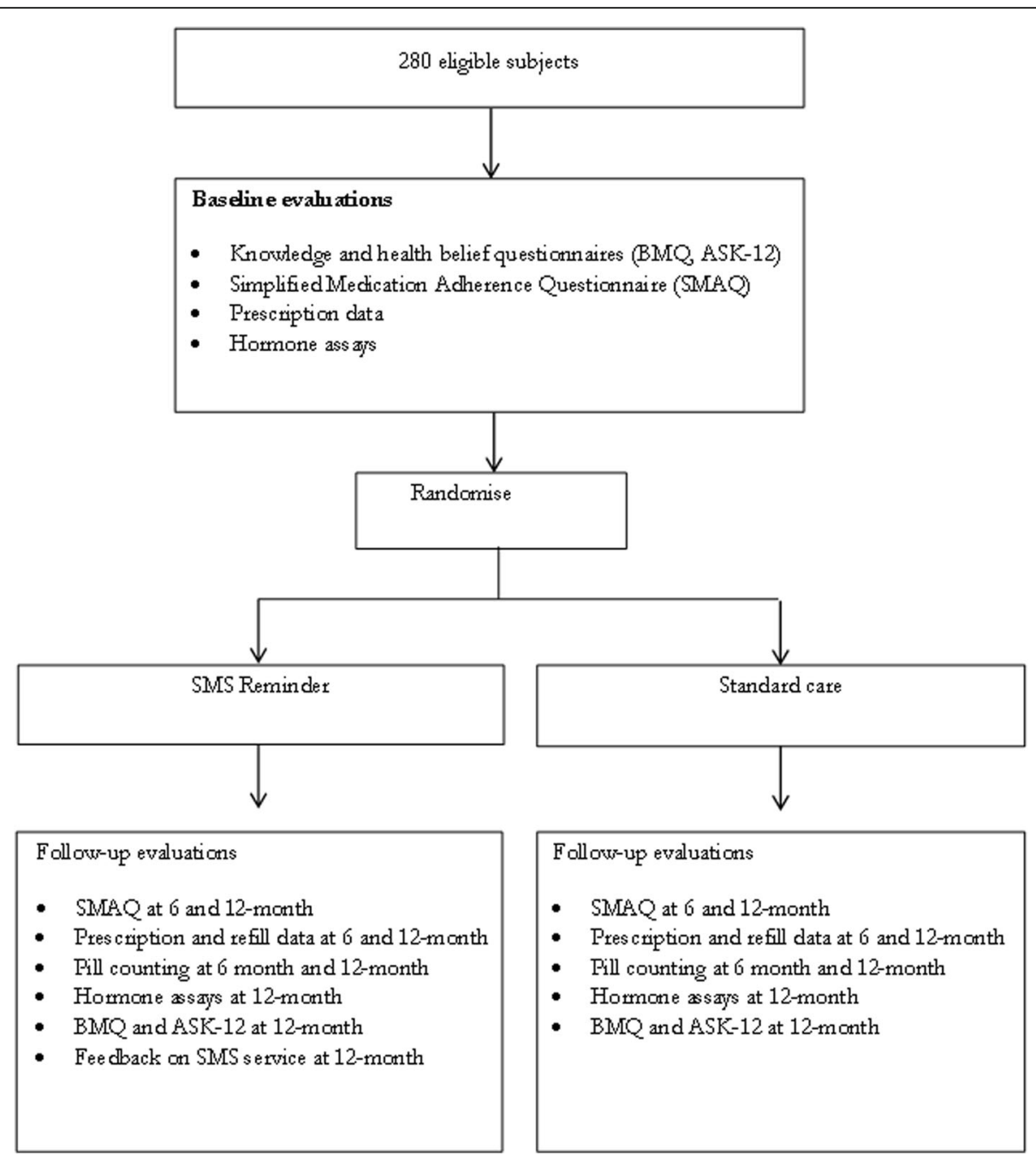

Fig. 2 Study flow chart

such as the sensitivity, specificity and kappa statistics using PDC as the gold standard. The percentages of adherence between the different measures will be compared via the McNemar's test.

\section{Ethics and dissemination}

Informed consent will be obtained by the co-Principal Investigators or research coordinator during the screening visit, prior to randomisation into the trial. The patient's consent to participate in the trial should be obtained after a full explanation has been given of the intervention options and the manner of intervention allocation. A copy of the signed consent form with study information will be given to the patients for their retention. The right of the patient to refuse to participate without giving reasons must be respected. After the patient has entered the trial, the clinician must remain free to give alternative intervention to that specified in the protocol at any stage if she feels it to be in the patient's best interest. The patient must remain free to withdraw at any time from the protocol intervention without giving reasons and without prejudicing her further treatment.

The protocol and the associated informed consent documents have been reviewed and approved by the institutional review board of both study sites. The results of this trial will be published in a peer-reviewed journal.

\section{Data quality assurance}

To ensure accuracy, completeness and reliability, the data will be prospectively collected and entered into the electronic database. Hardcopies of the data collection forms will be checked for completeness and verified by the research coordinator before data entry. Validity range checks will also be built into the database. At the study closure, $10 \%$ of the records will be randomly sampled for data quality assurance. 


\section{Confidentiality of data and patient records}

All data will be treated with strict confidentiality and will not be shared with parties other than members of the investigating team. All data will be centrally coordinated and the hardcopy forms will be kept under lock-and-key in a cabinet in the research coordinator's office. The softcopy data will be stored in a hard disk and protected by password. Subjects will be identified by a unique trial number and their identities will not be revealed in the data collection forms or questionnaires. A patient study ID list containing patient's name, personal identification number, mobile phone number and trial number will be maintained and kept in a study folder separate from the data collection forms.

\section{Discussion}

In recent years, various interventions have been utilised to improve medication adherence to AI therapy for breast or other types of cancer [37, 38]. A pilot randomised controlled trial of a web-enabled application to provide real-time monitoring and better management of treatment-related adverse symptoms among patients with hormone-receptor positive breast cancer showed web-enabled application with weekly reminders significantly improved short-term AI adherence. Also, several studies have suggested that short message reminder could benefit adherence to breast selfexamination or cancer-screening among breast cancer patients $[39,40]$. Chung et al. suggested that self-reported breast self-examination (BSE) adherence and the frequency of BSE were significantly higher in the intervention group than in the control group in the 6-month period [39]. However, to our knowledge, there has not been any study involving short-term clinical outcomes such as hormonal assays from reminders for breast cancer patients on AI therapies.

Studies on breast cancer have demonstrated that patients commonly reported suffering from physical and psychological inconvenience due to AI therapies [41]. As compared to women without a history of cancer, breast cancer patients, reported significantly higher odds of new onset of forgetfulness, difficulty in concentrating, hair loss and numbness or tingling in the extremities after the first 6 months of AI therapy, with odds ratio ranging between 2 to 4 folds [41]. A German study also showed that age contributed to the difference in medication adherence amongst breast cancer women who were treated with AI [42]. It suggested that patients aged over 70 years exhibited a $25 \%$ reduction in risk of treatment discontinuation as compared to younger patients. However, a literature review discussing poor adherence with hormonal therapy among women with breast cancer disagreed with the notion that non-adherence could be due to older age ( $\geq 55$ years). It reported that other reasons for hormonal therapy non-adherence could be due to patient's behaviour, treatment toxicity, cost of health care or medications, and comorbidities [43]. Also, it has been further suggested that medication compliance may be improved if financial barriers were removed [43]. Meanwhile, other researchers have identified socio-demographic and clinical characteristics to be associated with medication beliefs about AI such as concern, perceived necessity of taking the drug to improve one's health, and cancer/health worry amongst postmenopausal women [44].

This trial has several strengths as well as limitations. This will be the first study to evaluate short-term clinical outcomes from SMS reminder for breast cancer patients on AI therapy. Random allocation to SMS reminder or control arm ensures that patients in both arms will be comparable with respect to demographic and clinical characteristics, and any difference in outcomes can be attributed to the intervention. However, participants are not blinded to the assignment of intervention, thus there may be potential for bias in outcome assessments.

In short, sending SMS reminder to early-stage breast cancer women could be an added feature to existing healthcare protocol for breast cancer women if the results from our trial are promising. Understanding the potential barriers to medication adherence would enable policy makers to make informed decision when promoting regulations to minimise the impact of the barriers while formulating policies to elevate medication adherence.

\section{Abbreviations \\ Al: Aromatase inhibitor; ANCOVA: Analysis of covariance; ASK: Adherence Starts with Knowledge; BMQ: Beliefs about Medicines Questionnaire; BSE: Breast self-examination; ER: Estrogen receptor; NCIS: National University Cancer Institute, Singapore; PDC: Proportion of days covered; SMAQ: Simplified Medication Adherence Questionnaire; SMS: Short message service; UPIRTSO: Unanticipated problems involving risks to subjects and others}

Acknowledgements

The authors would like to thank the oncologists, nursing staff, research coordinators and patients at the participating sites for their contribution.

\begin{abstract}
Funding
This trial is supported by the Singapore Cancer Society Cancer Research Grant 2014; National University Cancer Institute, Singapore (NCIS) Centre Grant Seed Funding Program (Aug 2014 Grant Call); National University Health System Bridging Funds FY17. These funding sources had no role in the design of this study and will not have any role during its execution, analysis, interpretation of the data, or decision to submit results.
\end{abstract}

\section{Authors' contributions}

AW, CCT, PW, SCL and BCT participated in the design of the study and research protocol. EHT and $\mathrm{YXH}$ will coordinate the study, and are responsible for data acquisition. EHT, $\mathrm{YXH}$ and $\mathrm{BCT}$ will conduct statistical analysis. YXH, EHT, AW, CCT, PW, SCL and BCT were involved in the writing, editing, and approval of the final manuscript. All authors have read and approved the final manuscript. 


\section{Ethics approval and consent to participate}

The protocol (version 5, dated 24 April 2015) and the associated informed consent documents have been reviewed and approved by the National Healthcare Group Domain Specific Review Board on 4 May 2015, under the reference number DSRB 2014/01316. Protocol amendments, if any, will be reviewed and approved by the DSRB and documented in a memorandum. Written informed consent will be obtained from participants in this study.

\section{Consent for publication}

Not applicable.

\section{Competing interests}

The authors declare that they have no competing interests.

\section{Publisher's Note}

Springer Nature remains neutral with regard to jurisdictional claims in published maps and institutional affiliations.

\section{Author details}

'Saw Swee Hock School of Public Health, National University of Singapore and National University Health System, 12 Science Drive 2, \#10-03F, Singapore 117549, Singapore. ${ }^{2}$ Department of Haematology-Oncology, National University Cancer Institute, NUHS Tower Block Level 7, 1E Kent Ridge Road, Singapore 119228, Singapore. ${ }^{3}$ Department of General Surgery, Ng Teng Fong General Hospital, 1 Jurong East Street 21, Singapore 609606, Singapore. ${ }^{4}$ Division of Oncology Pharmacy, National University Cancer Institute, NUHS Tower Block Level 7, 1 E Kent Ridge Road, Singapore 119228, Singapore. ${ }^{5}$ Yong Loo Lin School of Medicine, National University of Singapore and National University Health System, 1E Kent Ridge Road, Singapore 119228, Singapore.

\section{Received: 7 February 2018 Accepted: 4 July 2018}

Published online: 09 July 2018

\section{References}

1. Delamater AM. Improving patient adherence. Clinical Diabetes. 2006;24:71-7.

2. Sabaté E. Adherence to long-term therapies : evidence for action. Geneva: World Health Organization; 2003. http://apps.who.int/iris/bitstream/10665/ 42682/1/9241545992.pdf. Accessed 1 Jan 2018

3. DiMatteo MR, Giordani PJ, Lepper HS, Croghan TW. Patient adherence and medical treatment outcomes: a meta-analysis. Med Care. 2002;40(9):794-811.

4. Howell A, Cuzick J, Baum M, Buzdar A, Dowsett M, Forbes JF, Hoctin-Boes G, Houghton J, Locker G, Tobias J. Results of the ATAC (Arimidex, tamoxifen, alone or in combination) trial after completion of 5 years' adjuvant treatment for breast cancer. Lancet. 2005;365(9453):60-2.

5. Ho PM, Bryson CL, Rumsfeld JS. Medication adherence: its importance in cardiovascular outcomes. Circulation. 2009:119(23):3028-35.

6. World Health Organization. Breast cancer: prevention and control. 2017 http://www.who.int/cancer/detection/breastcancer/en/index1.html. Accessed 1 Jan 2018

7. National Registry of Diseases Office. Singapore Cancer Registry Annual Registry Report. 2017.https://www.nrdo.gov.sg/docs/librariesprovider3/ Publications-Cancer/cancer-registry-annual-report-2015_web.pdf?sfvrsn=10. Accessed 18 Nov 2017.

8. Fabian CJ. The what, why and how of aromatase inhibitors: hormonal agents for treatment and prevention of breast cancer. Int J Clin Pract. 2007; 61(12):2051-63.

9. Partridge AH, LaFountain A, Mayer E, Taylor BS, Winer E, Asnis-Alibozek A. Adherence to initial adjuvant anastrozole therapy among women with early-stage breast cancer. J Clin Oncol. 2008;26:556-62.

10. Key T, Appleby P, Barnes I, Reeves G. Endogenous sex hormones and breast cancer in postmenopausal women: reanalysis of nine prospective studies. J Natl Cancer Inst. 2002;94(8):606-16.

11. Johnston SR, Dowsett M. Aromatase inhibitors for breast cancer: lessons from the laboratory. Nat Rev Cancer. 2003;3(11):821-31.

12. Cao A, Zhang J, Liu X, Wu W, Liu Y, Fan Z, Zhang A, Zhou T, Fu P, Wang S, et al. Health-related quality of life of postmenopausal Chinese women with hormone receptor-positive early breast cancer during treatment with adjuvant aromatase inhibitors: a prospective, multicenter, non-interventional study. Health Qual Life Outcomes. 2016;14:51.
13. Ryden L, Heibert Arnlind M, Vitols S, Hoistad M, Ahlgren J. Aromatase inhibitors alone or sequentially combined with tamoxifen in postmenopausal early breast cancer compared with tamoxifen or placebo meta-analyses on efficacy and adverse events based on randomized clinical trials. Breast. 2016;26:106-14.

14. Francis PA, Regan MM, Fleming GF, Láng I, Ciruelos E, Bellet M, Bonnefoi HR, Climent MA, Da Prada GA, Burstein HJ. Adjuvant ovarian suppression in premenopausal breast cancer. N Engl J Med. 2015;372(5):436-46.

15. Breastcancer.org. Aromatase Inhibitors. 2017. http://www.breastcancer.org/ treatment/hormonal/aromatase_inhibitors. Accessed 20 Jan 2018.

16. Murphy CC, Bartholomew LK, Carpentier MY, Bluethmann SM, Vernon SW. Adherence to adjuvant hormonal therapy among breast cancer survivors in clinical practice: a systematic review. Breast Cancer Res Treat. 2012;134(2): 459-78

17. Verma S, Madarnas Y, Sehdev S, Martin G, Bajcar J. Patient adherence to aromatase inhibitor treatment in the adjuvant setting. Curr Oncol. 2011; 18(Suppl 1):S3-9.

18. Valachis A, Garmo H, Weinman J, Fredriksson I, Ahlgren J, Sund M, Holmberg L. Effect of selective serotonin reuptake inhibitors use on endocrine therapy adherence and breast cancer mortality: a populationbased study. Breast Cancer Res Treat. 2016;159(2):293-303.

19. Costa E, Giardini A, Savin M, Menditto E, Lehane E, Laosa O, Pecorelli S, Monaco A, Marengoni A. Interventional tools to improve medication adherence: review of literature. Patient Prefer Adherence. 2015;9:1303-14.

20. Greer JA, Amoyal N, Nisotel L, Fishbein JN, MacDonald J, Stagl J, Lennes I, Temel JS, Safren SA, Pirl WF. A systematic review of adherence to oral antineoplastic therapies. Oncologist. 2016;21(3):354-76.

21. Australia and New Zealand Horizon Scanning Network. Horizon Scanning Technology Prioritising Summary- The use of SMS text messaging to improve outpatient attendance. 2007. http://www.horizonscanning.gov.au/ internet/horizon/publishing.nsf/Content/ 6B81AEB3E7EE0001CA2575AD0080F344/\$File/ May\%20Vol\%2016\%20No\%201\%20-\%20SMS.pdf. Accessed 1 Jan 2018.

22. Park LG, Howie-Esquivel J, Dracup K. A quantitative systematic review of the efficacy of mobile phone interventions to improve medication adherence. J Adv Nurs. 2014;70(9):1932-53.

23. Matthews K. Improving medication adherence for oncology patients through high-tech. Interventions. 2015; https://www. specialtypharmacytimes.com/publications/specialty-pharmacy-times/2015/ june-2015/improving-medication-adherence-for-oncology-patients-throughhigh-tech-interventions. Accessed 8 Jan 2018

24. Hanauer DA, Wentzell K, Laffel N, Laffel LM. Computerized automated reminder diabetes system (CARDS): E-mail and SMS cell phone text messaging reminders to support diabetes management. Diabetes Technol Ther. 2009:11(2):99-106.

25. Malcolm SE, Ng JJ, Rosen RK, Stone VE. An examination of HIV/AIDS patients who have excellent adherence to HAART. AIDS Care. 2003;15(2):251-61.

26. Molfenter TD, Bhattacharya A, Gustafson DH. The roles of past behavior and health beliefs in predicting medication adherence to a statin regimen. Patient Prefer Adherence. 2012;6:643-51.

27. Yang GS, Kim HJ, Griffith KA, Zhu S, Dorsey SG, Renn CL. Interventions for the treatment of aromatase inhibitor-associated arthralgia in breast cancer survivors: a systematic review and meta-analysis. Cancer Nurs. 2017:40(4): E26-41.

28. Neugut Al, Zhong X, Wright JD, Accordino M, Yang J, Hershman DL. Nonadherence to medications for chronic conditions and nonadherence to adjuvant hormonal therapy in women with breast cancer. JAMA Oncol. 2016;2(10):1326-32.

29. Bright EE, Petrie KJ, Partridge AH, Stanton AL. Barriers to and facilitative processes of endocrine therapy adherence among women with breast cancer. Breast Cancer Res Treat. 2016;158(2):243-51.

30. Hsieh K-P, Chen L-C, Cheung K-L, Chang C-S, Yang Y-H. Interruption and non-adherence to long-term adjuvant hormone therapy is associated with adverse survival outcome of breast cancer women-an Asian populationbased study. PLoS One. 2014;9(2):e87027.

31. Vervloet M, Linn AJ, van Weert JC, De Bakker DH, Bouvy ML, Van Dijk L. The effectiveness of interventions using electronic reminders to improve adherence to chronic medication: a systematic review of the literature. Am Med Inform Assoc. 2012;19(5):696-704.

32. Ortega Suárez FJ, Sánchez Plumed J, Pérez Valentín MA, Pereira Palomo P, Muñoz Cepeda MA, Lorenzo Aguiar D. Validation on the simplified 
medication adherence questionnaire (SMAQ) in renal transplant patients on tacrolimus. Nefrologia. 2011;31(6):690-6.

33. Horne R, Weinman J, Hankins M. The beliefs about medicines questionnaire: the development and evaluation of a new method for assessing the cognitive representation of medication. Psychol Health. 1999;14:1-24.

34. Matza LS, Park J, Coyne KS, Skinner EP, Malley KG, Wolever RQ. Derivation and validation of the ASK-12 adherence barrier survey. Ann Pharmacother. 2009:43(10):1621-30.

35. Garfield S, Clifford S, Eliasson L, Barber N, Willson A. Suitability of measures of self-reported medication adherence for routine clinical use : a systematic review. BMC Med Res Methodol. 2011;1:149.

36. Nau DP. Proportion of days covered (PDC) as a preferred method of measuring medication adherence. Springfield, VA: Pharmacy Quality Alliance; 2012. http://www.pqaalliance.org/images/uploads/files/ PQA\%20PDC\%20vs\%20\%20MPR.pdf. Accessed 22 Aug 2014

37. Graetz I, McKillop CN, Stepanski EJ, Vidal GA, Lee SS. Use of a web-based app to improve breast cancer symptom management and aromatase inhibitor adherence. J Clin Oncol. 2017;35(Suppl 5):89.

38. Fishbein JN, Nisotel LE, MacDonald JJ, Amoyal Pensak N, Jacobs JM, Flanagan C, Jethwani K, Greer JA. Mobile application to promote adherence to oral chemotherapy and symptom management: a protocol for design and development. JMIR Res Protoc. 2017;6(4):e62.

39. Chung IY, Kang E, Yom CK, Kim D, Sun Y, Hwang Y, Jang JY, Kim SW. Effect of short message service as a reminder on breast self-examination in breast cancer patients: a randomized controlled trial. J Telemed Telecare. 2015; 21(3):144-50.

40. Vidal C, Garcia M, Benito L, Milà N, Binefa G, Moreno V. Use of text-message reminders to improve participation in a population-based breast Cancer screening program. J Med Syst. 2014;38(9):118.

41. Gallicchio L, Calhoun C, Helzlsouer K. A prospective study of aromatase inhibitor therapy initiation and self-reported side effects. Support Care Cancer. 2017;25(9):2697-705.

42. Jacob L, Hadji P, Kostev K. Age-related differences in persistence in women with breast cancer treated with tamoxifen or aromatase inhibitors in Germany. J Geriatr Oncol. 2016;7(3):169-75.

43. Hershman DL. Sticking to it: improving outcomes by increasing adherence. J Clin Oncol. 2016:34(21):2440-2.

44. Salgado TM, Davis EJ, Farris KB, Fawaz S, Batra P, Henry NL. Identifying socio-demographic and clinical characteristics associated with medication beliefs about aromatase inhibitors among postmenopausal women with breast cancer. Breast Cancer Res Treat. 2017;163(2):311-9.

\section{Ready to submit your research? Choose BMC and benefit from:}

- fast, convenient online submission

- thorough peer review by experienced researchers in your field

- rapid publication on acceptance

- support for research data, including large and complex data types

- gold Open Access which fosters wider collaboration and increased citations - maximum visibility for your research: over $100 \mathrm{M}$ website views per year

At BMC, research is always in progress.

Learn more biomedcentral.com/submissions 